\title{
Computed tomography angiography as a confirmatory test for the diagnosis of brain death
}

\author{
Mark P. Garrett, MD, ${ }^{1}$ Richard W. Williamson, MD, ${ }^{1}$ Michael A. Bohl, MD, ${ }^{1}$ C. Roger Bird, MD, ${ }^{2}$ and \\ Nicholas Theodore, MD ${ }^{1}$
}

Departments of ${ }^{1}$ Neurosurgery and ${ }^{2}$ Neuroradiology, Barrow Neurological Institute, St. Joseph's Hospital and Medical Center, Phoenix, Arizona

\begin{abstract}
OBJECTIVE For a diagnosis of brain death (BD), ancillary testing is performed if patient factors prohibit a complete clinical examination and apnea test. The American Academy of Neurology (AAN) guidelines identify cerebral angiography (CA), cerebral scintigraphy, electroencephalography, and transcranial Doppler ultrasonography as accepted ancillary tests. CA is widely considered the gold standard of these, as it provides the most reliable assessment of intracranial blood flow. CT angiography (CTA) is a noninvasive and widely available study that is also capable of identifying absent or severely diminished intracranial blood flow, but it is not included among the AAN's accepted ancillary tests because of insufficient evidence demonstrating its reliability. The objective of this study was to assess the statistical performance of CTA in diagnosing BD, using clinical criteria alone or clinical criteria plus CA as the gold-standard comparisons.
\end{abstract}

METHODS The authors prospectively enrolled 22 adult patients undergoing workup for BD. All patients had cranial imaging and clinical examination results consistent with $\mathrm{BD}$. In patients who met the AAN clinical criteria for $\mathrm{BD}$, the authors performed CA and CTA so that both tests could be compared with the gold-standard clinical criteria. In cases that required ancillary testing, CA was performed as a confirmatory study, and CTA was then performed to compare against clinical criteria plus CA. Radiographic data were evaluated by an independent neuroradiologist. Test characteristics for CTA were calculated.

RESULTS Four patients could not complete the standard BD workup and were excluded from analysis. Of the remaining 18 patients, 16 met AAN criteria for BD, 9 of whom required ancillary testing with $C A$. Of the 16 patients, 2 who also required $C A$ ancillary testing were found to have persistent intracranial flow and were not declared brain dead at that time. These patients also underwent CTA; the results were concordant with the CA results. Six patients who were diagnosed with $\mathrm{BD}$ on the basis of clinical criteria alone also underwent CA, with $100 \%$ sensitivity. For all 18 patients included in the study, CTA had a sensitivity of $75 \%$, a specificity of $100 \%$, a positive predictive value of $100 \%$, and a negative predictive value of $33 \%$.

CONCLUSIONS Clinical examination with or without CA remains the gold standard in BD testing. Studies assessing the statistical performance of CTA in BD testing should compare CTA to these gold standards. The statistical performance of CTA in BD testing is comparable to several of the nationally accepted ancillary tests. These data add to the growing medical literature supporting the use of CTA as a reliable ancillary test in BD testing.

https://thejns.org/doi/abs/10.3171/2016.10.JNS161042

KEY WORDS brain death; cerebral angiography; computed tomography angiography; diagnostic technique

I N 1968, an ad hoc committee at the Harvard Medical School published a set of criteria that sought to define the clinical state of brain death (BD). ${ }^{4}$ Since that time, the concepts and methods for determining BD continue to be refined. In an effort to create a legal definition for BD in the United States, the 1993 President's Commission wrote the Uniform Determination of Death Act, which defined death as either 1) the irreversible cessation of circulatory and respiratory functions, or 2) the irreversible cessation of all functions of the entire brain, including the brainstem. ${ }^{16}$ Although the commission stipulated that the determination of death must be made with accepted medical standards, it did not define those standards. In 1995, the American Academy of Neurology (AAN) published a set

ABBREVIATIONS AAN = American Academy of Neurology; $B D=$ brain death; $C A=$ cerebral angiography; $C T A=C T$ angiography; $E E G=$ electroencephalography; $I C A=$ internal carotid artery; ICV = internal cerebral vein; $M C A=$ middle cerebral artery; NMPT = nuclear medicine perfusion test; TCD = transcranial Doppler ultrasonography; VA = vertebral artery.

SUBMITTED April 22, 2016. ACCEPTED October 21, 2016.

INCLUDE WHEN CITING Published online March 17, 2017; DOI: 10.3171/2016.10.JNS161042. 
of criteria that are widely regarded as the standard for the clinical determination of BD. ${ }^{11}$ These criteria have proven to be reliable and, in most cases, allow the practitioner to confidently declare a patient brain dead by clinical criteria alone.22

Over time, confirmatory tests for brain function have been developed that provide additional data in situations with confounding factors. These tests can be grouped into 2 broad categories: 1) those that examine the electrical activity of the brain, and 2) those that assess the cerebral circulation. Among these ancillary studies, cerebral angiography (CA) has long been considered the gold standard, based on the theory that cerebral circulatory arrest is incompatible with life and that such arrest is most reliably diagnosed with CA. Newer tests, such as CT angiography (CTA), offer noninvasive methods for obtaining the same information. Multiple studies have attempted to show the reliability of CTA in demonstrating a lack of intracranial blood flow in the brain-dead patient. - $3,5-10,12-15,17-19$ However, these studies are highly heterogeneous in terms of the comparison studies they use as gold standards; some appropriately use clinical criteria alone, whereas others use a combination of clinical criteria and any one or a combination of the AAN-recommended ancillary tests. The use of ancillary tests other than CA as gold standards to compare with CTA is problematic, as many of these studies have a statistical performance similar to that of CTA..$^{18}$ Although accumulating data support the use of CTA as a reliable ancillary test, there is no consensus regarding the protocols for administering the test or interpreting the results. ${ }^{9}$ This is particularly true in the United States where nuclear medicine studies and CA are more commonly used to confirm a diagnosis of BD.

In our own practice, we rely almost entirely on clinical criteria alone to declare $\mathrm{BD}$, and we reserve the use of ancillary tests for situations in which confounding factors complicate clinical assessment. In these cases, CTA offers certain advantages over other studies, because it is widely available and can be completed quickly. However, before CTA is accepted nationally as a reliable ancillary test, further studies are needed to compare CTA against the current gold standards in BD diagnosis: clinical criteria alone, or clinical criteria plus CA in cases with confounders. We therefore analyzed the performance of CTA against these gold standards for the diagnosis of BD.

\section{Methods \\ Patient Population}

From October 2009 until June 2012, all adult patients admitted to our institution who underwent workup for BD were recruited to be part of this study. Eligible patients were adults (age $\geq 18$ years) who had given an appropriate medical power of attorney to a person who could provide consent on their behalf. All patients had an irreversible and proximate cause of their poor neurological status. Baseline data recorded for each patient included age, sex, urine toxicology results, baseline creatinine, and mechanism of brain injury. The study was approved by the institutional review board of St. Joseph's Hospital and Medical Center, Phoenix, Arizona.

\section{Clinical Determination of BD}

All patients were evaluated by the neurosurgical service for the determination of $\mathrm{BD}$, which included cranial imaging. After reversible causes of coma were ruled out, patients underwent a thorough neurological examination. Neurological assessments supporting the diagnosis of BD were performed according to the 2010 AAN guidelines. ${ }^{22}$ All patients with clinical examination results consistent with BD then underwent an apnea test. Patients who completed a proper apnea test and did not show any signs of spontaneous respiration were then diagnosed with $\mathrm{BD}$, and subsequently underwent both CA and CTA testing to compare the results of these studies with the clinical diagnosis. Patients who could not complete a proper apnea test underwent $\mathrm{CA}$ as part of their formal diagnostic workup for $\mathrm{BD}$, which was then followed by CTA for comparison with the clinical examination and CA as the confirmatory study.

\section{Radiographic Evaluation}

CA was performed using a dedicated biplane angiographic system (Axiom Artis, Siemens Healthcare). The femoral artery was punctured in a retrograde fashion, and a 5-Fr introducer sheath (Cook Medical) was inserted. A 5-Fr catheter (Cordis Corp.) was used for selective catheterization of the common carotid and vertebral arteries (VAs). Contrast (Isovue-300, Bracco Diagnostics Inc.) was injected with a power injector (Medrad Inc.). Injection rates were typically $8 \mathrm{ml} / \mathrm{sec}$, for a total of $10 \mathrm{ml}$ in the common carotid arteries, and $5 \mathrm{ml} / \mathrm{sec}$, for a total of 8 $\mathrm{ml}$ in the VAs. CA consisted of anteroposterior and lateral views of the head with each injection. Imaging parameters included a $1024 \times 1024$ matrix and a field of view of 22 $\mathrm{cm}$. The filming rate was 3 images $/ \mathrm{sec}$ for a total of $20-30$ seconds.

After CA, patients underwent scanning using a LightSpeed VCT CT scanner (General Electric Co.). The protocol consisted of unenhanced CT of the head, CTA of the head after injection of $50 \mathrm{ml}$ of Omnipaque 300 (GE Healthcare Inc.) into an antecubital vein at $4 \mathrm{ml} / \mathrm{sec}$, and a 60-second delayed CTA of the head. Axial, sagittal, and coronal maximum intensity projection images were also obtained in the CTA data sets.

Both CA and CTA studies were then interpreted by a single neuroradiologist (C.R.B.) in a nonblinded manner. We first analyzed a direct comparison of blood flow between CA and CTA. In each study, contrast opacification was analyzed for 12 different vessels. The vessels that were analyzed included the external carotid artery, the internal carotid artery (ICA), the 3 segments of the anterior cerebral artery $\left(\mathrm{A}_{1}, \mathrm{~A}_{2}\right.$, and $\left.\mathrm{A}_{3}\right)$, the 3 segments of the middle cerebral artery (MCA; $\mathrm{M}_{1}, \mathrm{M}_{2}$, and $\mathrm{M}_{3}$ ), the VA, the basilar artery, the posterior cerebral artery, and the internal cerebral veins (ICVs). The radiographic findings on $\mathrm{CA}$ that were considered to be consistent with BD were absence of contrast flow above the level of the petrous ICAs and intradural VAs, as well as absence of flow in the ICVs. Using CTA, we then applied the French 4-vessel opacification criteria for BD confirmation (Table 1). ${ }^{6}$

Serum creatinine was measured both before and after radiographic imaging to assess the effect of contrast load on renal function. 
TABLE 1. Angiographic criteria for brain death

Angiography

Absence of flow above the petrous ICA

Absence of flow in the intradural VA

Absence of flow in the ICV

$\mathrm{CTA}^{*}$

Absence of flow in the right cortical MCAs $\left(M_{3}\right)$

Absence of flow in the left cortical MCAs $\left(M_{3}\right)$

Absence of flow in the right ICV

Absence of flow in the left ICV

${ }^{*}$ Criteria from Frampas et al. ${ }^{6}$

\section{Data Analysis}

All data were collected prospectively. The paired-sample t-test was used to compare pre- and posttest creatinine levels after both confirmatory tests were performed. Results are presented as numbers and percentages (mean \pm $\mathrm{SD})$. A $\mathrm{p}$ value $<0.05$ was considered significant.

\section{Results}

\section{Demographics}

Twenty-two adult patients ( 9 women and 13 men) were ultimately enrolled during the study period. The mean age of the patients was $47 \pm 13.2$ years. One-half $(50 \%$; $n=$ 11) of the 22 patients were declared brain dead after either hemorrhagic $(n=9)$ or ischemic $(n=2)$ cerebrovascular accidents (Table 2). Four patients were excluded from

TABLE 2. Demographics of 22 adult patients with suspected brain death

\begin{tabular}{cccl}
\hline Case No. & Age (yrs) & Sex & \multicolumn{1}{c}{ Diagnosis at Presentation } \\
\hline 1 & 48 & F & Hemorrhagic cerebrovascular accident \\
\hline 2 & 22 & M & Gunshot wound \\
\hline 3 & 37 & M & Traumatic brain injury \\
\hline 4 & 57 & F & Subarachnoid hemorrhage \\
\hline 5 & 49 & F & Hemorrhagic cerebrovascular accident \\
\hline 6 & 44 & M & Hemorrhagic cerebrovascular accident \\
\hline 7 & 42 & F & Hemorrhagic cerebrovascular accident \\
\hline 8 & 53 & M & Hemorrhagic cerebrovascular accident \\
\hline 9 & 46 & F & Traumatic brain injury \\
\hline 10 & 67 & M & Hemorrhagic cerebrovascular accident \\
\hline 11 & 42 & F & Subarachnoid hemorrhage \\
\hline 12 & 48 & F & Subarachnoid hemorrhage \\
\hline 13 & 29 & M & Subarachnoid hemorrhage \\
\hline 14 & 64 & F & Ischemic cerebrovascular accident \\
\hline 15 & 48 & M & Ischemic cerebrovascular accident \\
\hline 16 & 24 & M & Subarachnoid hemorrhage \\
\hline 17 & 61 & M & Hemorrhagic cerebrovascular accident \\
\hline 18 & 50 & M & Hemorrhagic cerebrovascular accident \\
\hline 19 & 66 & M & Traumatic brain injury \\
\hline 20 & 22 & M & Traumatic brain injury \\
\hline 21 & 54 & F & Hemorrhagic cerebrovascular accident \\
\hline 22 & 53 & M & Subarachnoid hemorrhage \\
\hline & & &
\end{tabular}
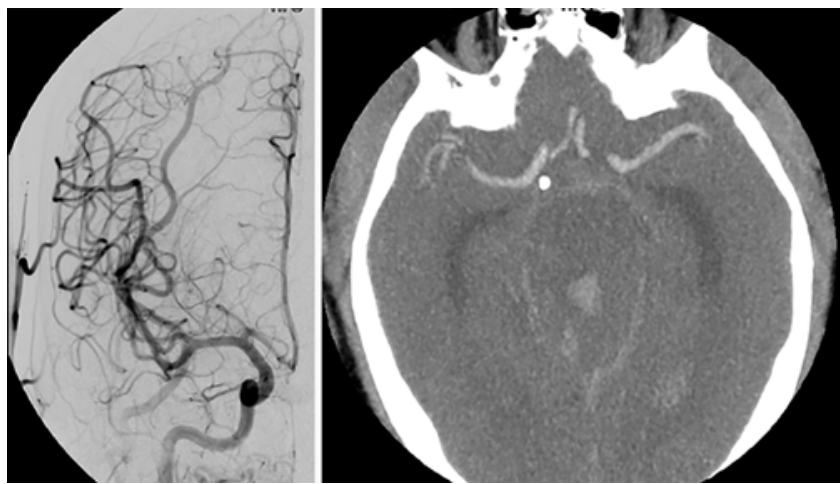

FIG. 1. Patient with apnea test aborted because of unstable oxygen saturation. CA image (left) and CTA image (right) showing persistent intracranial flow. The family of the patient elected to pursue palliative measures, and the patient died soon thereafter.

analysis because formal diagnostic workup for BD could not be completed.

Of the remaining 18 patients, $16(89 \%)$ met AAN criteria for $\mathrm{BD}$. Nine $(56 \%)$ of these 16 patients required ancillary testing with CA due to positive urine toxicology results for neurodepressants $(\mathrm{n}=8)$ or cardiac instability during apnea testing $(\mathrm{n}=1)$. The 2 patients who also required CA ancillary testing because they did not meet the AAN criteria were found to have persistent intracranial flow and thus were not declared brain dead at that time (Figs. 1 and 2). These patients also underwent CTA, the findings of which were concordant with the CA results. Seventeen patients underwent both CA and CTA. One patient who met the clinical criteria for BD did not undergo CA because of cardiac arrest shortly after CTA. The mean time between the 2 tests was $78.5 \pm 59.1$ minutes. Figure 3 summarizes the categorization of the 22 patients.

\section{Radiographic Data}

Of the 18 patients analyzed with CTA, 16 (89\%) were diagnosed with BD by AAN criteria. Twelve $(75 \%)$ of these 16 patients had CTA findings that were positive for BD. The 2 patients who did not meet BD criteria had CTA findings that were negative for BD. CTA had a sensitivity

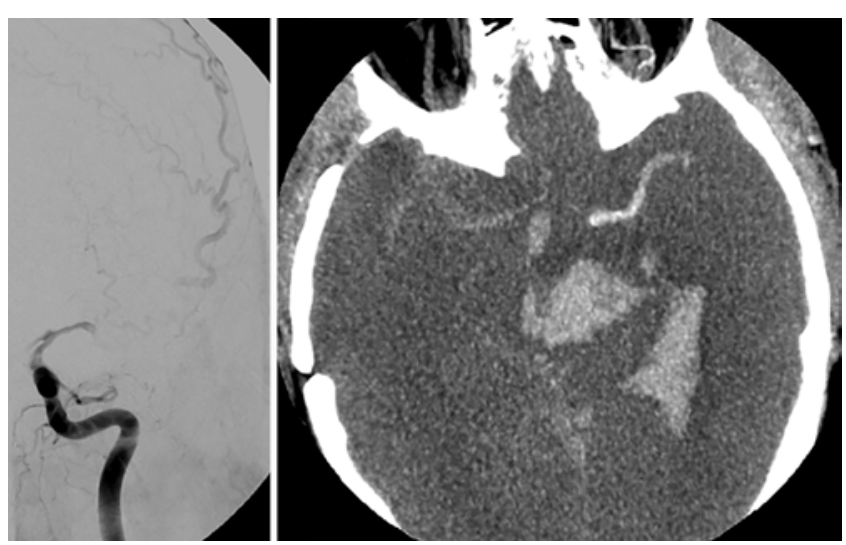

FIG. 2. Patient with positive apnea test but persistent flow in $M_{1}$, the left segment of the MCA. CA image (left) and CTA image (right) showing concordant findings. 


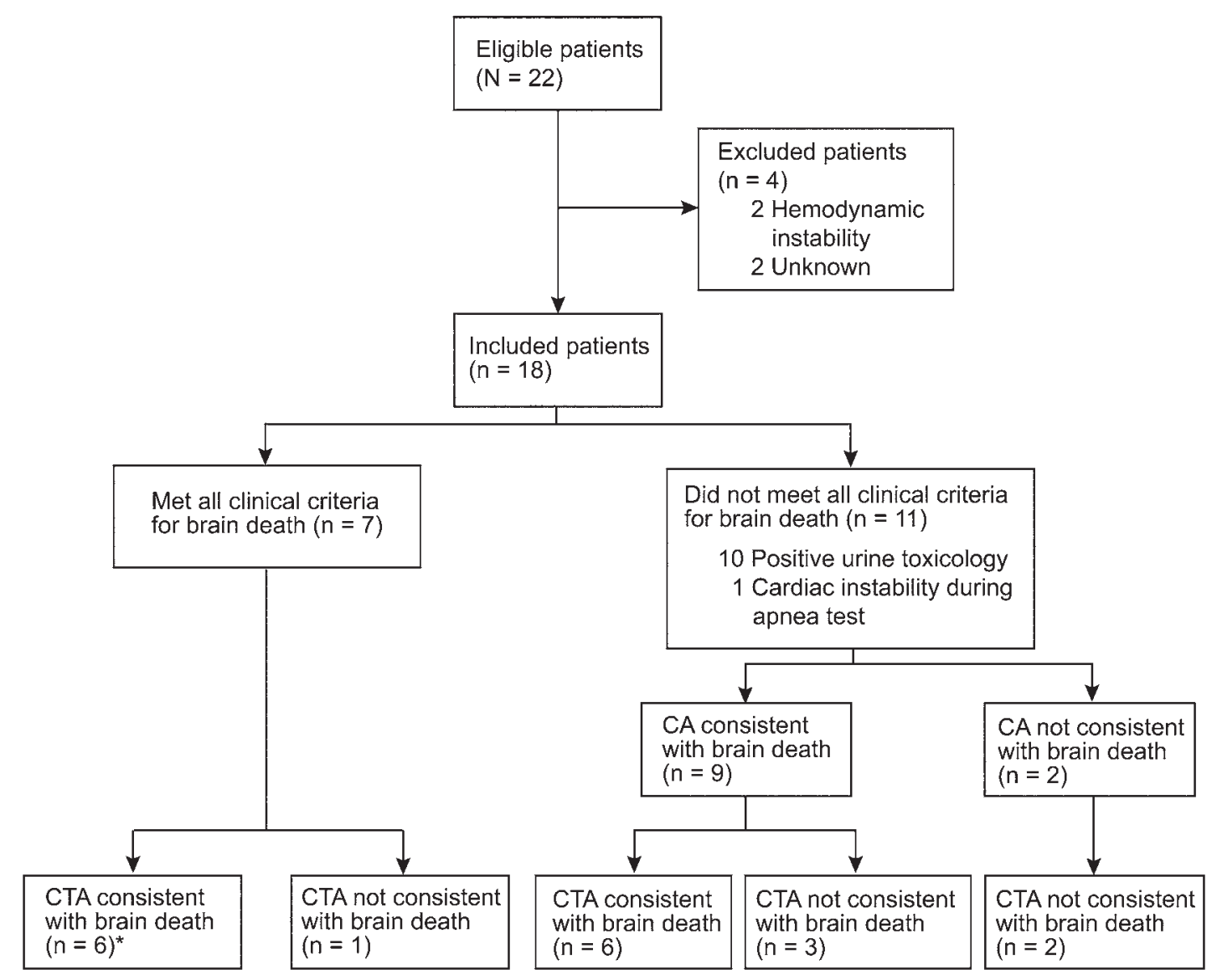

FIG. 3. Diagnostic categorization of enrolled patients. ${ }^{*}$ One patient did not undergo CA due to cardiac arrest following CTA.

of $75 \%$, a specificity of $100 \%$, a positive predictive value of $100 \%$, and a negative predictive value of $33 \%$ (Table 3 ). Six patients were diagnosed with BD on the basis of clinical criteria alone and also had CA. All 6 of these patients had $\mathrm{CA}$ results consistent with $\mathrm{BD}$.

\section{Laboratory Data}

Laboratory data were available for 16 patients who underwent both confirmatory tests. Creatinine values were recorded before and after each test. There were no adverse events related to contrast administration with either test. The mean serum creatinine was $1.82 \pm 2.51 \mathrm{mg} / \mathrm{dl}$ before both tests and $2.42 \pm 3.44 \mathrm{mg} / \mathrm{dl}$ after both tests. The mean change in creatinine for these patients was $0.538 \pm 1.41$ $\mathrm{mg} / \mathrm{dl}$. There was no significant difference found in the pre- and posttest serum creatinine after both tests were performed $(\mathrm{p}=0.14)$.

\section{Discussion}

The diagnosis of BD is principally a clinical one, but ancillary tests must often be used when certain components of the clinical assessment are incomplete or unclear. In its 1995 practice parameters, the AAN listed 5 ancillary tests that were considered acceptable when clinical assessment alone was insufficient for diagnosing BD, ${ }^{11}$ and these tests are still cited by some authors as the most appropri- ate assessments to be used when making a BD diagnosis.? These tests are CA, electroencephalography (EEG), transcranial Doppler ultrasonography (TCD), nuclear medicine perfusion test (NMPT), and somatosensory evoked potentials. In his background paper for the 1995 AAN practice parameters, Wijdicks ${ }^{20}$ identified several shortcomings in these tests: lack of interobserver studies and no interpretation guidelines for CA; poor sensitivity and reliability of EEG; lack of experience with NMPTs; and variable interpretations of TCD results, depending on the pathology, location, and skill of the ultrasonographer. This list of accepted studies has since become dated because some of the tests are no longer commonly used in clinical practice. In countries such as France and Canada, for example, newer national guidelines no longer support the use of EEG or other electrophysiological tests, but instead deem CTA and other tests of cerebral perfusion to be appropriate ancillary tests. ${ }^{10}$ Arguments have also recently been made in Germany for the inclusion of CTA in their national guidelines for the diagnosis of BD. ${ }^{18}$

In its 2010 evidence-based guidelines update, the AAN stated that there was insufficient evidence to determine whether CTA accurately confirms the cessation of function of the entire brain, so CTA could not be considered at that time as an acceptable ancillary test. ${ }^{22}$ Interestingly, the AAN still included EEG as one of the 4 preferred tests (NMPT, TCD, and CA being the others) despite the well- 
TABLE 3. CTA statistical performance

\begin{tabular}{lccc}
\hline CTA Result & $\begin{array}{c}\text { Gold Standard: } \\
\text { BD Positive }\end{array}$ & $\begin{array}{c}\text { Gold Standard: } \\
\text { BD Negative }\end{array}$ & $\begin{array}{c}\text { Total No. } \\
\text { of Patients }\end{array}$ \\
\hline CTA positive for BD & 12 & 0 & 12 \\
\hline CTA negative for BD & 4 & 2 & 6 \\
\hline Total no. of patients & 16 & 2 & 18 \\
\hline
\end{tabular}

known shortcomings in this test's ability to confirm cessation of function of the entire brain and brainstem, because it only tests cortical function and so is inherently incapable of confirming the cessation of deep cortical and brainstem function. Further complicating matters, pathological studies have shown that in many patients who meet the clinical criteria for $\mathrm{BD}$, significant areas of viable brain tissue can often be found, challenging the maxim that any test of $\mathrm{BD}$, clinical or otherwise, must prove cessation of function of the entire brain and brainstem..$^{21}$ Therefore, a truly worthwhile ancillary test is one with maximum specificity such that any positive result would indicate such extensive central nervous system damage that BD could be unequivocally determined. As the 2008 Canadian expert report on BD concluded, cerebral perfusion tests are best suited for diagnosing $\mathrm{BD}$, as cerebral circulatory arrest is most clearly incompatible with neuronal function. ${ }^{13}$ Because tests of cerebral perfusion such as CTA are actually tests of cerebral circulatory arrest, these are the most appropriate tests to use when attempting to clarify an ambiguous diagnosis of $\mathrm{BD}$, as they tend to have the smallest falsepositive rates. ${ }^{2,14,18}$ Our data support these claims, as CTA maintained a specificity of $100 \%$ and a sensitivity of $75 \%$ in our test population. Test sensitivity in this context refers to the number of patients who meet the gold-standard criteria for BD but have CTA findings that do not meet the stated criteria for BD (opacification above the level of the petrous ICAs, intradural VAs, or ICVs). Our 75\% sensitivity and $33 \%$ negative predictive value likely can be explained by artifactual flow, or "stasis filling," of the intracranial vessels. Although this relatively high false-negative rate is suboptimal, in the context of BD diagnosis, it is far more preferable to a test with poor specificity, which could potentially lead one to diagnose BD in a patient who is not brain dead.

This prospective assessment of CTA contributes to the growing medical literature supporting the inclusion of CTA among the accepted ancillary tests for diagnosis of BD. Although our small sample size prohibits broader conclusions regarding the statistical performance of CTA as a test for BD, our results are consistent with those previously reported by others. ${ }^{9,15}$ Furthermore, the statistical performance of CTA reported in the literature is similar to that of EEG and TCD, 2 AAN-approved ancillary tests. ${ }^{2,9,14,15,18}$

Numerous radiological criteria exist for interpreting CTA results in patients suspected of being brain dead, with widely varying sensitivities resulting from how many and which vessels are included in a given criteria. In 2009, Frampas et al. ${ }^{6}$ compared the standard French 7-vessel criteria (nonopacification of pericallosal and cortical segments of the MCAs, ICVs, and great cerebral vein) with a 4-vessel criteria (nonopacification of cortical segments of MCAs and ICVs) and found that, by using the 4-vessel criteria, sensitivity was improved from $62.8 \%$ to $85.7 \%$ while maintaining a specificity of $100 \%$. Furthermore, SuarezKelly et al. ${ }^{14}$ found that performing quantitative analysis of vessel opacity on CTA could increase the sensitivity of CTA to $97 \%$ while maintaining $100 \%$ specificity. Other authors have attempted to further elucidate the sensitivity and specificity of CTA by comparing it to TCD and EEG..$^{12,19}$ The results of such studies are highly variable and offer little meaningful data in the assessment of CTA, because the comparison tests themselves had similar statistical performance and validity to CTA for diagnosis of BD. ${ }^{18}$ The only meaningful comparisons in this field are those made to the widely accepted gold standards: clinical examination, or clinical examination plus CA when confounders are present.

\section{Study Limitations and Future Directions}

Our findings are limited by all the limitations inherent in a small, single-institution study. The small number of patients $(n=22)$ recruited over the study period may suggest a selection bias; however, our experience was simply that it is difficult to obtain consent from families for participation in a clinical trial while they are still acutely grieving the loss of their loved one. The small number of patients analyzed also limits the applicability of our results to a larger population. Despite these shortcomings, we believe that, when considered within the context of the larger and growing literature on the use of CTA in BD diagnosis, our results meaningfully support claims that CTA is an appropriate ancillary test.

Our results also demonstrated an increase in creatinine following angiographic studies. Although this increase was not found to be statistically significant, it should be noted that this result may ultimately be shown to be significant in a larger study. Although acute kidney injury is likely to be present in many patients undergoing workup for $\mathrm{BD}$, the effect of contrast on renal function should not be overlooked when considering an ancillary test for the diagnosis of $\mathrm{BD}$.

Future studies on the use of CTA should ascribe to a uniform set of criteria and gold-standard comparisons. We recommend the French 4-vessel opacification system as the standard diagnostic criterion, and the clinical examination with or without CA as the gold-standard comparison. The application of these criteria in future studies should result in the accrual of a sufficient quality and quantity of data in the literature to allow an accurate assessment of CTA for diagnosis of BD. Although recent meta-analyses are mixed in their recommendations regarding the use of CTA as an ancillary test, ${ }^{9,15}$ we believe that our findings, in addition to those in the current literature, support the continued use of CTA as a confirmatory test in any circumstance in which the clinical diagnosis of $\mathrm{BD}$ is incomplete or unclear.

\section{Conclusions}

In this small prospective study, CTA was a reliable ancillary test in patients who did not meet all clinical criteria for a diagnosis of $\mathrm{BD}$. These data add to the growing inter- 
national literature demonstrating the reliability of CTA for diagnosis of cerebral circulatory arrest, as its sensitivity is comparable to that of other AAN-approved ancillary tests and its specificity is close to $100 \%$. Our results thus provide a compelling argument for the inclusion of CTA as an acceptable confirmatory test in future national guidelines on $\mathrm{BD}$ determination.

\section{References}

1. Bohatyrewicz R, Sawicki M, Walecka A, Walecki J, Rowinski O, Bohatyrewicz A, et al: Computed tomographic angiography and perfusion in the diagnosis of brain death. Transplant Proc 42:3941-3946, 2010 (Erratum in Transplant Proc 43:668, 2011)

2. Brasil S, Bor-Seng-Shu E, de-Lima-Oliveira M, Azevedo MK, Teixeira MJ, Bernardo L, et al: Role of computed tomography angiography and perfusion tomography in diagnosing brain death: a systematic review. J Neuroradiol 43:133-140, 2016

3. Combes JC, Chomel A, Ricolfi F, d'Athis P, Freysz M: Reliability of computed tomographic angiography in the diagnosis of brain death. Transplant Proc 39:16-20, 2007

4. Committee of the Harvard Medical School to Examine the Definition of Brain Death: a definition of irreversible coma. JAMA 205:337-340, 1968

5. Dupas B, Gayet-Delacroix M, Villers D, Antonioli D, Veccherini MF, Soulillou JP: Diagnosis of brain death using two-phase spiral CT. AJNR Am J Neuroradiol 19:641-647, 1998

6. Frampas E, Videcoq M, de Kerviler E, Ricolfi F, Kuoch V, Mourey F, et al: CT angiography for brain death diagnosis. AJNR Am J Neuroradiol 30:1566-1570, 2009

7. Greer DM, Strozyk D, Schwamm LH: False positive CT angiography in brain death. Neurocrit Care 11:272-275, 2009

8. Heran MK, Heran NS, Shemie SD: A review of ancillary tests in evaluating brain death. Can J Neurol Sci 35:409419, 2008

9. Kramer AH, Roberts DJ: Computed tomography angiography in the diagnosis of brain death: a systematic review and metaanalysis. Neurocrit Care 21:539-550, 2014

10. Leclerc X: [CT angiography for the diagnosis of brain death: recommendations of the French Society of Neuroradiology (SFNR).] J Neuroradiol 34:217-219, 2007 (Fr)

11. Quality Standards Subcommittee of the American Academy of Neurology: Practice parameters for determining brain death in adults (summary statement). Neurology 45:10121014,1995

12. Quesnel C, Fulgencio JP, Adrie C, Marro B, Payen L, Lembert N, et al: Limitations of computed tomographic angiography in the diagnosis of brain death. Intensive Care Med 33:2129-2135, 2007

13. Shemie SD, Lee D, Sharpe M, Tampieri D, Young B: Brain blood flow in the neurological determination of death: Canadian expert report. Can J Neurol Sci 35:140-145, 2008

14. Suarez-Kelly LP, Patel DA, Britt PM, Clayton EJ, Bromberg WJ, McCain CM, et al: Dead or alive? New confirmatory test using quantitative analysis of computed tomographic angiography. J Trauma Acute Care Surg 79:995-1003, 2015

15. Taylor T, Dineen RA, Gardiner DC, Buss CH, Howatson A, Pace NL: Computed tomography (CT) angiography for confirmation of the clinical diagnosis of brain death. Cochrane Database Syst Rev 3:CD009694, 2014

16. Uniform Determination of Death Act, 12 Uniform Laws Annotated (U.L.A.) 589 (West 1993 and West Supp. 1997)

17. Welschehold S, Boor S, Reuland K, Beyer C, Kerz T, Reuland A, et al: CT angiography as a confirmatory test in brain death. Acta Neurochir Suppl 114:311-316, 2012

18. Welschehold S, Boor S, Reuland K, Thömke F, Kerz T, Reuland $\mathrm{A}$, et al: Technical aids in the diagnosis of brain death: a comparison of SEP, AEP, EEG, TCD and CT angiography. Dtsch Arztebl Int 109:624-630, 2012

19. Welschehold S, Kerz T, Boor S, Reuland K, Thömke F, Reuland A, et al: Computed tomographic angiography as a useful adjunct in the diagnosis of brain death. J Trauma Acute Care Surg 74:1279-1285, 2013

20. Wijdicks EF: Determining brain death in adults. Neurology 45:1003-1011, 1995

21. Wijdicks EF, Pfeifer EA: Neuropathology of brain death in the modern transplant era. Neurology 70:1234-1237, 2008

22. Wijdicks EF, Varelas PN, Gronseth GS, Greer DM: Evidence-based guideline update: determining brain death in adults: report of the Quality Standards Subcommittee of the American Academy of Neurology. Neurology 74:1911-1918, 2010

\section{Disclosures}

The authors report no conflict of interest concerning the materials or methods used in this study or the findings specified in this paper.

\section{Author Contributions}

Conception and design: Theodore, Garrett, Bird. Acquisition of data: Theodore, Garrett, Williamson. Analysis and interpretation of data: all authors. Drafting the article: Garrett, Williamson, Bohl. Critically revising the article: all authors. Reviewed submitted version of manuscript: Theodore, Garrett, Williamson, Bohl. Statistical analysis: Williamson, Bohl. Administrative/technical/ material support: all authors. Study supervision: Theodore.

\section{Correspondence}

Nicholas Theodore, c/o Neuroscience Publications, Barrow Neurological Institute, St. Joseph's Hospital and Medical Center, $350 \mathrm{~W}$ Thomas Rd., Phoenix, AZ 85013. email: neuropub@dignityhealth. org. 